\title{
Bayesian Approach to Condition Monitoring of PRC Bridges
}

\author{
Daniele Zonta ${ }^{1, a}$, Matteo Pozzi ${ }^{1, b}$ and Paolo Zanon ${ }^{1, c}$ \\ ${ }^{1}$ DIMS, University of Trento, Via Mesiano 77, 38100 Trento, Italy \\ adaniele.zonta@unitn.it, ’bmatteo.pozzi@unitn.it, cpaolo.zanon@unitn.it
}

Keywords: Damage detection, Bayesian updating, Monte Carlo simulation, Model selection, PRC bridges.

\begin{abstract}
This paper presents a damage detection procedure based on Bayesian analysis of data recorded by permanent monitoring systems as applied to condition assessment of Precast Reinforced Concrete (PRC) bridges. The concept is to assume a set of possible condition states of the structure, including an intact condition and various combinations of damage, such as failure of strands, cover spalling and cracking. Based on these states, a set of potential time response scenarios is evaluated first, each described by a vector of random parameters and by a theoretical model. Given the prior distribution of this vector, the method assigns posterior probability to each scenario as well as updated probability distributions to each parameter. The effectiveness of this method is illustrated as applied to a short span PRC bridge, which is currently in the design phase and will be instrumented with a number of fiber-optic long gauge-length strain sensors. A Finite Element Model is used to simulate the instantaneous and time-dependent behavior of the structure, while Monte Carlo simulations are performed to numerically evaluate the evidence functions necessary for implementation of the method. The ability of the method to recognize damage is discussed.
\end{abstract}

\section{Introduction}

We are seeing rapid development of sensor technology which will radically change monitoring methods for civil structures in coming years. Fiber optics technology offers today durable solutions for bridge monitoring, and recent advances in Micro Opto Electro Mechanical Systems (MOEMS) suggest that in the near future we will be able to rely on very small-scale optical devices. Wireless communication too can simplify many installation and operation issues. We also expect these technologies to be available at very low cost.

The possibility of monitoring bridge structures using a large number of sensors is very promising for the future of instrumental monitoring. However, a major issue is how to exploit appropriately the large amount of data recorded by these systems. A Bayesian approach provides a rational framework to interpret the data, also allowing proper handling of all prior knowledge, including material properties, environmental conditions and sensor performance. This methodology lets us identify not only the most likely values of the unknown damage parameters (such as type, position and extent) but also their posterior probability distribution. Bayesian theory of probability originates from Bayes' well known essay [1]. Many modern specialised textbooks provide the reader with a critical review and applications of this theory to data analysis (see for instance [2,3]). Of all the papers dealing with application of Bayesian theory to engineering problems, the authors wish to mention Beck's work $[4,5,6]$, which by disseminating these concepts had great impact on the civil engineering community.

In this paper we introduce an iterative damage detection algorithm based on Bayesian analysis as specifically applied to the problem of condition monitoring of Precast Reinforced Concrete (PRC) bridges. In the following section we will state the basic assumptions and the general formulation of the method. Next a sample application is presented to clarify how the method works: the case study is a single-span PRC box-girder bridge, adopted as reference in a research project aiming at developing a new concept of smart elements [7]; due to page limitation, the application is restricted to the simulation of failure of one prestressing strand. A brief discussion of the identification outcomes is provided at the end of the paper. 


\section{Identification Concept}

Problem statement. Assume we have a bridge instrumented with a certain number of sensors, and we want to gain information on the state of the bridge based on the data recorded. Each sensor provides a measurement for each of $N T$ time values $\left(t_{1}, t_{2}, \ldots, t_{N t}\right)$. It is convenient to distinguish two types of gauges: sensors recording the structural response of the bridge, and sensors recording the load and environmental effects on the bridge. The first set might include sensors such as strain gauges, accelerometers, displacement transducers. In a broad sense, we can classify as a response sensor any instrumentation setup capable of providing a response quantity. For simplicity, here we will assume that these are all strain gauges. Say that the structure is instrumented with $N s$ sensors of this type, labelled $\left(s_{1}, s_{2}, \ldots, s_{N s}\right)$, and let us label $\varepsilon_{i, j}$ the strain measurement recorded by sensor $s_{j}$ at time $t_{i}$. The second set includes, for example, thermocouples installed next to the strain gauges for temperature compensation or load cells applied at the bridge bearing, capable of recording the traffic loads. Without losing generality, here again we will assume all these sensors to be thermometers. The basic idea is that response measurements depend, on the one hand, on external actions such as temperature and loads; on the other on long term effects, such as dead load redistribution, creep, shrinkage, strand relaxation. Long term effects produce slow changes in the structural response. Based on this, it is convenient to organize measurements into time intervals (for example: per day) this time interval being a time span short enough to assume that long term changes are negligible, and long enough to ensure that short term change are significant. Let us define $\mathbf{m}_{T, j}$ the vector including all the strain measurements recorded by the $j$-th sensor in time interval $T$ and $\mathbf{m}_{T}$ the matrix including all strains in time interval $T$. Finally let us label $\mathbf{M}_{T}$ the whole dataset from the first time interval (i.e. from the start of monitoring) to time interval $T$. Similarly to strain measurements, we define as $\mathbf{h}_{T}$ the matrix including all temperature measurements in time interval $T$. Data acquired during this sample period can be organized in matrix form:

$$
\mathbf{m}_{T, j}=\varepsilon_{T, j}^{0}+\mathbf{h}_{T} \mathbf{a}_{T, i}+\mathbf{g}_{T, j}
$$

where $\varepsilon_{T, j}^{0}$ is strain independent of temperature (i.e. the compensated strain), $\mathbf{a}_{T, i}$ is the vector including the coefficients of the linear correlation from temperature to strain, and $\mathbf{g}_{T, j}$ is a noise vector, which is assumed to have zero mean Gaussian distribution, with standard deviation $\left(\sigma_{g}\right)_{T, j}$. Eq. 1 assumes that the effect of temperature (or in general of external actions) on strain is linear, although this is not the most general case.

We assume that the presence of damage in the structure will generally modify the compensated response history. Thus, the identification method seeks to detect the presence of damage by comparison of the compensated measurements with the theoretical response produced by a model. In practice, it is convenient to divide the domain of the possible structural response in a mutually exclusive and exhaustive set of scenarios $\left(\mathrm{S}_{1}, \mathrm{~S}_{2}, \ldots, \mathrm{S}_{N d}\right)$, each defining the structural behavior in a specific condition (e.g. reinforcement corrosion). A single probability result for each scenario is one of the main advantages of this approach. The structural strain response ${ }^{n} r_{T, j}\left({ }^{n} \mathbf{x}\right)$ for day $T$ and sensor $s_{j}$ in scenario $n$ is controlled by a certain number of parameters ${ }^{n} \mathbf{x}$ (e.g. damage position, activation time, corrosion rate). The structural response is completely defined by specifying a scenario and a value for the correlated parameter set. Here, as the Bayesian model selection theory [2,8], the discrete meta-parameter scenario identifies the response function which in turn is specified by a parameter set. The difference between measurements and structural response is just random noise. Assuming scenario $n$ and ${ }^{n} \mathbf{x}$ to be correct, the compensated strain can be expressed as $\varepsilon_{T, j}^{0}{ }^{n} r_{T, j}\left({ }^{n} \mathbf{x}\right)+e_{T, j}$, where $e_{T, j}$ is the model error. We assume $e_{T, j}$ is a random error modelled as an uncorrelated Gaussian noise, with zero mean and standard deviation $\left({ }^{n} \sigma_{e}\right)_{j}$. This is independent of time, but generally changes with the sensor. Evidently $\left({ }^{n} \sigma_{e}\right)_{j}$ changes with sensor type, but we may expect a dependency, for example, on sensor position or precision. More generally we can state that $\left({ }^{n} \sigma_{e}\right)_{j}$ is function of a set of scenario-dependent parameters ${ }^{n} \mathbf{y}$. In summary, we can observe that each scenario is fully described by a set of parameters ${ }^{n} \mathbf{p}=\left[{ }^{n} \mathbf{x}^{n} \mathbf{y}\right]$. 
Bayesian updating. The method here presented allows calculation of the probability of being in each scenario, as well as the statistical distribution of the parameters associated. Once the probability at day (or time interval) $T-1$ of being in a specific scenario is known, Bayes' theorem allows us to update this probability using the fresh data acquired on day (or time interval) $T$ :

$$
\operatorname{prob}\left(S_{n} \mid \mathbf{M}_{T}, I\right)=\frac{\operatorname{PDF}\left(\mathbf{m}_{T} \mid \mathbf{M}_{T-1}, S_{n}, I\right) \cdot \operatorname{prob}\left(S_{n} \mid \mathbf{M}_{T-1}, I\right)}{\operatorname{PDF}\left(\mathbf{M}_{T} \mid \mathbf{M}_{T-1}, I\right)} .
$$

where $I$ means all the background information assumed, and PDF stands for Probability Density Function. The first term at the numerator, sometimes referred to as evidence of scenario $S_{n}$, can be calculated integrating over the whole parameter domain $D^{n} \mathbf{p}$, using:

$$
\operatorname{PDF}\left(\mathbf{m}_{T} \mid \mathbf{M}_{T-1}, S_{n}, I\right)=\int_{D^{n} \mathbf{p}} \operatorname{PDF}\left(\mathbf{m}_{T} \mid{ }^{n} \mathbf{p}, S_{n}, I\right) \cdot \operatorname{PDF}\left({ }^{n} \mathbf{p} \mid \mathbf{M}_{T-1}, S_{n}, I\right) \cdot d^{n} \mathbf{p} .
$$

while the denominator of Eq. 2 can be expressed as:

$$
\operatorname{PDF}\left(\mathbf{M}_{T} \mid \mathbf{M}_{T-1}, I\right)=\sum_{n=1}^{N d} \operatorname{PDF}\left(\mathbf{m}_{T} \mid \mathbf{M}_{T-1}, S_{n}, I\right) \cdot \operatorname{prob}\left(S_{n} \mid \mathbf{M}_{T-1}, I\right) \text {. }
$$

In a similar manner, we can calculate the PDF of the parameters governing a specific scenario:

$$
\operatorname{PDF}\left({ }^{n} \mathbf{p} \mid \mathbf{M}_{T}, S_{n}, I\right)=\frac{\operatorname{PDF}\left(\mathbf{m}_{T} \mid{ }^{n} \mathbf{p}, S_{n}, I\right) \cdot \operatorname{PDF}\left({ }^{n} \mathbf{p} \mid \mathbf{M}_{T-1}, S_{n}, I\right)}{\operatorname{PDF}\left(\mathbf{m}_{T} \mid \mathbf{M}_{T-1}, S_{n}, I\right)} .
$$

where:

$$
\operatorname{PDF}\left(\left.\mathbf{m}_{T}\right|^{n} \mathbf{p}, S_{n}, I\right)=\prod_{j=1}^{N s} \operatorname{PDF}\left(\mathbf{m}_{T, j} \mid{ }^{n} \mathbf{p}, S_{n}, I\right) .
$$

Of course, the first application of Eqs. 2 and 5 requires definition of prior values of probability of each scenario $\operatorname{prob}\left(S_{n} \mid I\right)$ and Probability Density Function (PDF) of parameters vector $\operatorname{PDF}\left({ }^{n} \mathbf{p} \mid S_{n}, I\right)$. The only remaining problem is how to obtain $\operatorname{PDF}\left(\left.\mathbf{m}_{T, j}\right|^{n} \mathbf{p}, S_{n}, I\right)$. Assuming $\operatorname{PDF}\left(\mathbf{a}_{T, j} \mid I\right)$ to be Gaussian, it is demonstrated that:

$$
\operatorname{PDF}\left(\left.\mathbf{m}_{T, j}\right|^{n} \mathbf{p}, S_{n}, I\right)=\operatorname{Normal}\left({ }^{n} r_{T, j}\left({ }^{n} \mathbf{x}\right) ;\left(\mu_{\varepsilon}\right)_{T, j},\left(\sigma_{\varepsilon}\right)_{T, j}{ }^{2}+\left({ }^{n} \sigma_{e}\right)_{j}^{2}\right) \text {. }
$$

where $\left(\mu_{\varepsilon}\right)_{T, j}$ and $\left(\sigma_{\varepsilon}\right)_{T, j}$ are the mean value and standard deviation estimated for the compensated strain $\varepsilon_{T, j}^{0}$ introduced in Eq. 1. In turn, $\left(\mu_{\varepsilon}\right)_{T, j}$ and $\left(\sigma_{\varepsilon}\right)_{T, j}$ can be estimated using the following procedure: Eq 1 can be seen expressed in the set of parameters $\mathbf{v}_{T, j}=\left[\mathbf{a}_{T, j}{ }^{\mathrm{T}} \varepsilon^{0}{ }_{T, j}\right]^{\mathrm{T}}$; the best fit of $\mathbf{v}_{T, j}$ is first calculated; next, the error variance $\left(\sigma_{g}\right)_{T, j}$ is estimated comparing measurements and best fit; based on this, the covariance matrix of $\mathbf{v}_{T, j}$ is calculated; accounting for the prior distribution of $\mathbf{a}_{T, j}$ Bayes' theorem provides the posterior distribution of $\mathbf{v}_{T, j}$; finally, $\left(\mu_{\varepsilon}\right)_{T, j}$ and $\left(\sigma_{\varepsilon}\right)_{T, j}$ result from the marginalization of the posterior PDF of $\mathbf{v}_{T}$, which is a trivial procedure for Gaussian distributions.

Numerical methods for calculating evidence. When many parameters are involved in Eq. 3, the exact integration might require an exceptional computational effort and needs to be circumvented with numerical techniques. One of the simplest ways is to apply Laplace asymptotic expansion [4]: using this method, function $\operatorname{PDF}\left(\left.\mathbf{m}_{T}\right|^{n} \mathbf{p}, S_{n}, I\right) \cdot \operatorname{PDF}\left({ }^{n} \mathbf{p} \mid S_{n}, I\right)$ is approximated by an unnormalized multivariate Gaussian function. Algorithms of the Markov Chain Monte Carlo family are alternate methods for solving Eq. 4. Parallel Tempering [2] is one of the most robust options of this type: this is an evolution of the Metropolis-Hastings algorithm [5,9] where several pseudo-random samples are produced in parallel by using different distributions. The algorithm allows information exchange between the simulations running in parallel [2]. This approach is computationally more demanding but its validity is more general, as it also works with non-Gaussian distributions. 

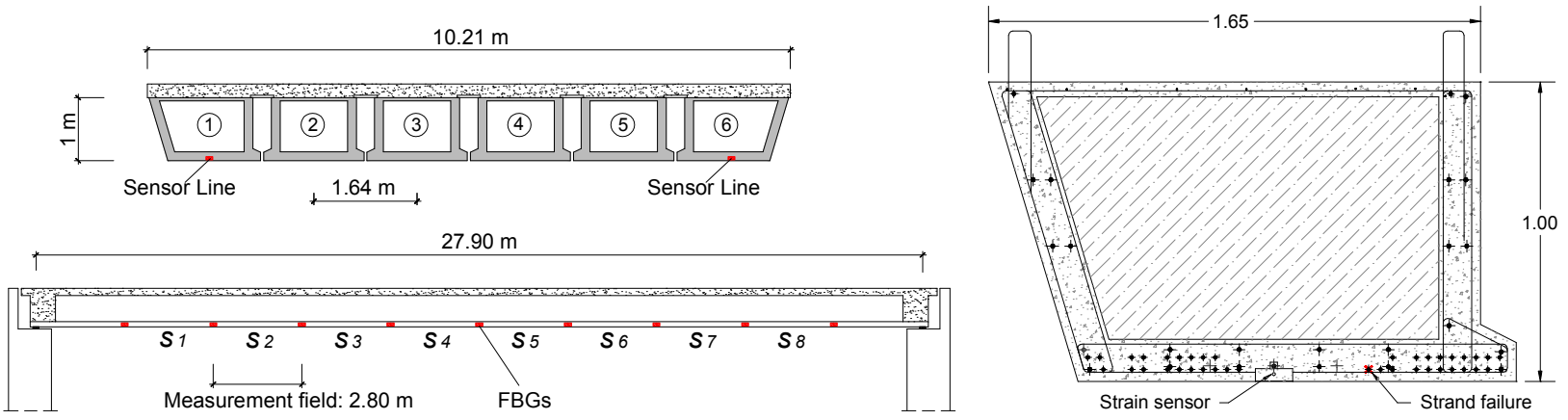

Fig. 1. Elevation and cross-section of the case study (left); cross-section of one of an instrumented box-girder (right); based on the original design by Autonomous Province of Trento and Rivoli SpA.

\section{Sample application}

Case study description. To clarify how the Bayesian updating procedure works, in the following we present the application of this method to a real-life problem. The case study is the PRC bridge shown in Fig. 1. The bridge has a single span of $27.9 \mathrm{~m}$ and deck $10.4 \mathrm{~m}$ width overall. The deck structure is made up of 6 precast elements, finished with a $22 \mathrm{~cm}$ cast-in-place concrete slab. The prefabricated elements are 1-m high box girders, prestressed with 400.6 " diameter strands. The two lateral beams are special smart elements specifically designed for this application with embedded instrumentation. In detail, each smart element accommodates 8 long gauge-length fiber optic sensors at the lower edge, each monitoring longitudinal elongation over a $2.8 \mathrm{~m}$ span. The optical instrumentation also includes thermometers for temperature compensation. The reader is referred to [7] for more information on the bridge design and the smart element technology involved.

Damage simulation. In this example, we simulate the failure of a strand located at beam 1 and position $x_{f}=15.1 \mathrm{~m}$ from the left hand support, near sensor $s_{4}$; we also suppose the failure occurs at time $t_{f}=43$ days after the production of the beam. To predict the strain history and distribution, we developed a Finite Element Model (FEM) of the deck accounting for all geometrical and mechanical properties of structural elements, including pre-stressing, creep, shrinkage and relaxation [10]. Assuming Young's modulus $E_{c}=35 \mathrm{GPa}$ and a creep coefficient $\phi=3.55$ for concrete, the FEM shows that the damage episode produces an instantaneous change in the strain field as reported in Fig. 2(a). Note that the local strain peak is $14 \mu \varepsilon$, which is relatively small with respect to long term effects, as highlighted in [7]. Suppose the monitoring system evaluates compensated strains with a sample period of one day, producing a sequence of measurements $\mathbf{M}_{T}$ from $T=1$ day to $T=51$ days: the theoretical compensated strain history obtained by FEM are of the type shown as the dashed line in Fig. 2(b) in the case of sensor $s_{4}$. However, in real life the compensated response is affected by instrumental error, imprecise compensation of external action and other unpredictable factors. To account for all these uncertainties a Gaussian noise with standard deviation of $15 \mu \varepsilon$ was added the pure FEM response. The resulting signals are similar to those shown as a continuous line in Fig. 2(b).

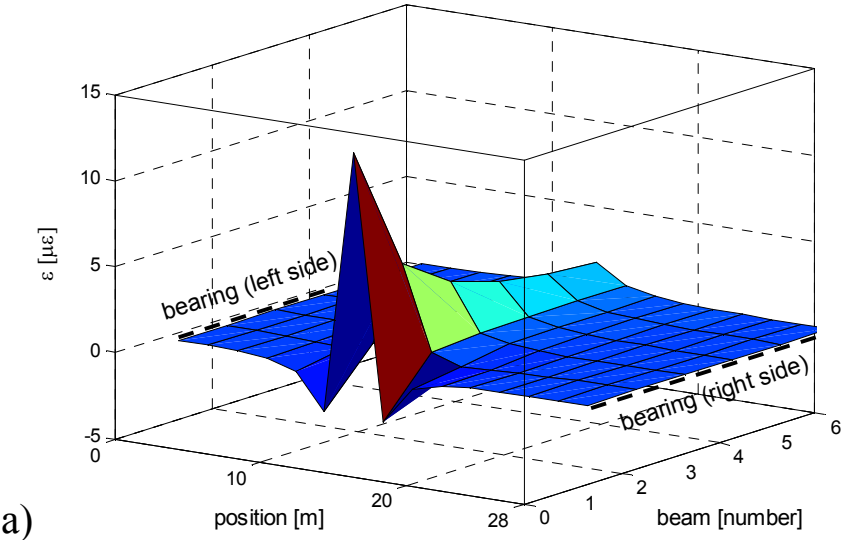

b)

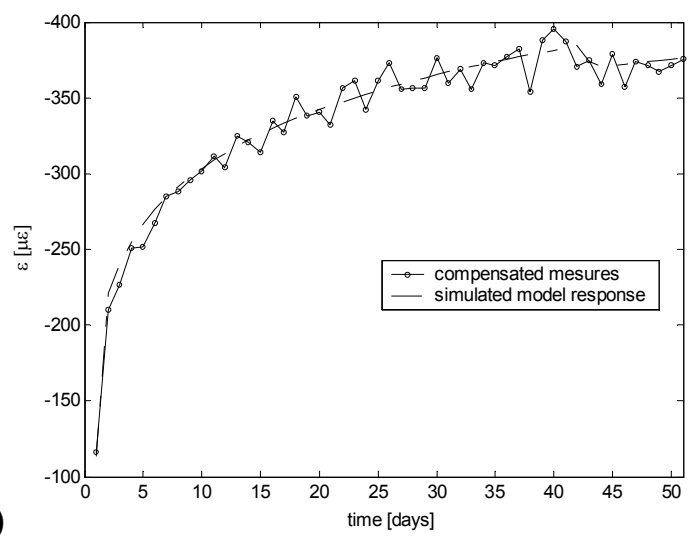

Fig. 2. Changes in the strain field due to the failure of a strand on beam 1 (a). Simulation of the compensated strain response recorded at sensor $s_{4}$ on beam 1 . 
Damage identification. Our objective is now to recognize the presence and position of damage, based on the simulated sensor response. To do so, we assume that only two scenarios, $S_{1}$ and $S_{2}$, are allowed: $S_{1}$ simulates a situation where no damage occurs and the structural response follows a trend basically due to creep; $S_{2}$ simulates a strand failure scenario. Given that the strain sensors are all the same type, we can assume that $\left({ }^{n} \sigma_{e}\right)_{j}$ is independent of the sensor, thus the set of parameter ${ }^{n} \mathbf{y}$ reduces to a single standard deviation ${ }^{n} \sigma_{e}$. Therefore, scenarios $S_{1}$ and $S_{2}$ are characterized by response parameters ${ }^{l} \mathbf{p}=\left[\begin{array}{lll}{ }^{l} \mathrm{Ec} & { }^{l} \phi & { }^{l} \sigma_{e}\end{array}\right]$ and ${ }^{2} \mathbf{p}=\left[{ }^{2} \mathrm{Ec}{ }^{2} \phi t_{f} x_{f}{ }^{2} \sigma_{e}\right]$, respectively. A priori, a total ignorance is assumed between scenarios, i.e. $\operatorname{prob}\left(S_{1} \mid I\right)=\operatorname{prob}\left(S_{2} \mid I\right)=0.5$, and the parameters are assumed uncorrelated. In detail: $\operatorname{PDF}\left({ }^{1} E_{c} \mid I\right)$ and $\operatorname{PDF}\left({ }^{2} E_{c} \mid I\right)$ are both Gaussian with mean value $\mu_{E c}=40 \mathrm{GPa}$ and standard deviation $\sigma_{E c}=10 \mathrm{GPa}$; similarly, $\operatorname{PDF}\left({ }^{1} \phi \mid I\right)$ and $\operatorname{PDF}\left({ }^{2} \phi \mid I\right)$ are Gaussian with mean value $\mu_{\phi}=$ 3 and standard deviation $\sigma_{\phi}=1$; uniform PDF is assumed for $\operatorname{PDF}\left(\ln \left({ }^{1} \sigma_{e}\right) \mid I\right)=\operatorname{PDF}\left(\ln \left({ }^{2} \sigma_{e}\right) \mid I\right)$ between $1 \mu \varepsilon$ and $30 \mu \varepsilon$, and uniform distributions are also assumed for $t_{f}$ and $y_{f}$. In both scenarios, the structural response is reproduced using the same FEM adopted in simulating the sensor response.

In general, the probability of being in a specific scenario and the PDFs of the corresponding parameters are calculated day by day through the recursive application of Eqs. 2 and 5. For this specific problem, the special nature of time variable $t_{f}$ lets us simplify the burdensome numerical calculation of evidence in scenario $S_{2}$. First note that for $t_{f}>T$ evidently we have that ${ }^{l} r_{T, s}\left({ }^{l} \mathbf{x}\right) \equiv$ ${ }^{2} r_{T, s}\left({ }^{2} \mathbf{x}\right)$, thus the posterior PDF for ${ }^{l} \mathbf{x}$ at time $T-1$ can be used as prior PDF for the corresponding parameters in ${ }^{2} \mathbf{x}$ for step $T-1$ to $T$. Next, the problem simplifies significantly observing that after a few days monitoring the distribution of parameters ${ }^{l} \mathbf{x}$ basically remains almost unchanged. In practice, the numerical procedure here adopted follows two parallel paths: when data from a new time interval $T$ is available, on the one hand $\operatorname{PDF}\left({ }^{l} \mathbf{p} \mid \mathbf{M}_{T}, S_{I}, I\right)$ is updated by Parallel Tempering; on the other, evidence of $S_{2}$ is estimated by direct integration over the two damage parameters, $t_{f}$ and $x_{f}$, and treating $E c, \phi$ and $\sigma_{e}$ with Laplace approximation.

Fig. 3(a) shows the result of the Parallel Tempering procedure for strain data at $T=41$ days in the space $E c-\phi$. The simulation starts from the centre of prior PDF, and after a few steps converges to the bulk of posterior $\operatorname{PDF}\left({ }^{1} \mathbf{x} \mid \mathbf{M}_{41}, S_{1}, I\right)$. This distribution is closely approximated by a Gaussian PDF with $\mu_{E c}=35.10 \mathrm{GPa}, \mu_{\phi}=3.565$, thus very close to the actual value adopted in the simulation, and marginal standard deviations $\sigma_{E c}=0.15 \mathrm{GPa}$ and $\sigma_{\phi}=0.039$. At $T=41$ days, the probability $\operatorname{prob}\left(S_{2} \mid \mathbf{M}_{41}, I\right)$ of damage is actually very close to zero. This value departs from zero only a few measurements after the actual occurrence of damage; and at the end of the simulated monitoring period, $T=51$ days, we obtain $\operatorname{prob}\left(S_{2} \mid \mathbf{M}_{51}, I\right)=0.703$. Fig. 3(b) shows the joint distribution of the two damage parameters, time of failure $t_{f}$ and position $x_{f}$, at $T=51$ days. It is worth noting that the procedure identifies the damage position very well, while time of occurrence is more uncertain, with the most likely value for $t_{f}=45$ days.

a)

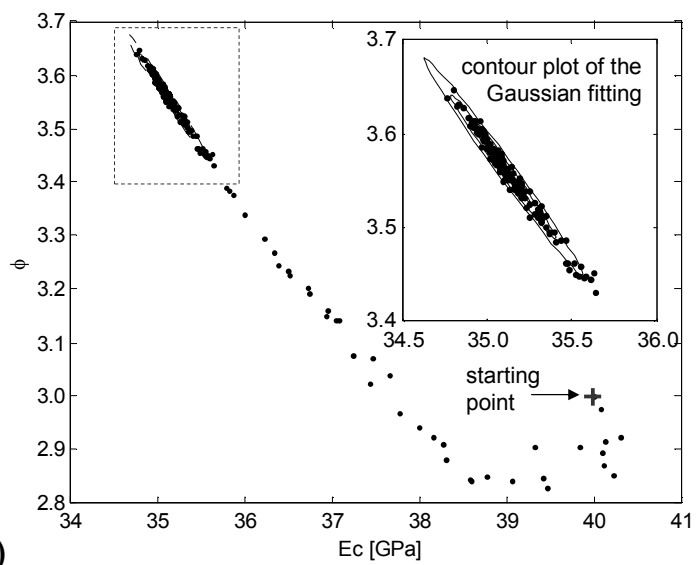

Fig. 3. Random walk of Parallel Tempering simulation in $E c-\phi$ space in scenario $S_{l}$ in time span 1 to 41 days (a). Marginal PDF of damage parameters $t_{f}$ and $x_{f}$ at time $T=51$ days (b). 


\section{Summary}

The proposed identification procedure provides a rational quantification of the influence of monitoring data on the knowledge of the occurrence of different scenarios. With respect to classical damage detection methods, its merit is to provide not only information on the damage, but also the degree of confidence of this information. This is of paramount importance when the results of damage assessment serve as an input in a decision-making process. For instance, the simulation reported clearly shows that parameters such as mechanical properties of material and damage position can be identified with very high precision. However, the specific nature of the problem does not allow us to recognize with the same confidence the time of occurrence of damage.

The procedure has been developed with in mind the problem of monitoring PRC bridges, and also the example proposed is extremely simplified. However, the general approach is not problem dependent, and can be extended to a broader class of problems, including manifold scenarios, model or material uncertainties, prior knowledge of parameter distribution. The main issue is the high computational cost involved in the integration of evidences, especially when, as in this case, the response model is based on a FEM.

\section{Acknowledgements}

The project mentioned in this paper is partially funded by the Italian Earthquake Engineering Laboratory Network (RELUIS), and involves contributions by the Autonomous Province of Trento (PAT) and Rivoli SpA, of Rivoli Veronese. The analysis presented is based on the preliminary design project provided by the Autonomous Province of Trento and by Rivoli SpA. The box beam element models presented in this paper are offered on catalog by Rivoli SpA. The authors wish also to thank Matteo Faccincane for his contribution to the finite element analysis.

\section{References}

[1] T. Bayes: Philos. Trans. R. Soc. London, Vol. 53 (1763), p. 370.

[2] P. Gregory: Bayesian Logical Data Analysis for the Physical Sciences (Cambridge University Press, Cambridge, UK, 2005).

[3] D.S. Sivia: Data Analysis: a Bayesian Tutorial (Oxford University Press, Oxford, UK 2006).

[4] C. Papadimitriou, J.L. Beck and L.S. Katafyogiotis: J. Eng. Mech. Vol. 123(12) (1997), p. 380.

[5] J.L. Beck and S.-K. Au: J. Eng. Mech. Vol. 128(4) (2002), p. 380.

[6] J.L. Beck and L.S. Katafygiotis: J. Eng. Mech. Vol. 124(2) (1998), p. 455.

[7] D. Zonta, M. Pozzi, A. Colombo and O.S. Bursi: Smart Structures and Materials 2006: Smart Sensor Monitoring Systems and Applications. SPIE proc. Vol. 6167 (2006), p. 131.

[8] G.L. Bretthorst, in: Maximum Entropy and Bayesian Methods, edited by G.R. Heidbreder, Springer New York, N.Y. (1996).

[9] N. Metropolis, A. Rosenbluth, M. Rosenbluth, A. Teller and E. Teller: J. of Chemical Physics Vol. 21 (1953), p. 1087.

[10] M.P. Collins, D. Mitchell: Prestressed Concrete Structures (Prentice Hall, Englewood Cliffs, New Jersey, 1991). 